\title{
Aprimoramento de mecanismo dosador de rebolos para o plantio mecanizado de cana-de-açúcar ${ }^{1}$
}

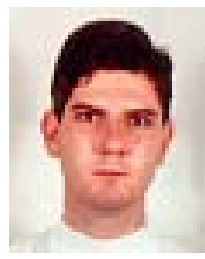

Américo F. Dias Neto², Paulo S. G. Magalhães ${ }^{3} \&$ Oscar A. Braunbeck ${ }^{4}$

\author{
1 Parte da Dissertação de Mestrado do primeiro autor \\ 2 FEAGRI/UNICAMP. E-mail: americo@agr.unicamp.br (Foto) \\ ${ }^{3}$ FEAGRI/UNICAMP. Cid. Univ. Zeferino Vaz, CP6011, CEP 13083-970. Fone: (19) 3788-1053. E-mail: paulo@agr.unicamp.br \\ 4 FEAGRI/UNICAMP. Fone: (19) 3788-1049. E-mail: oscar@agr.unicamp.br
}

Protocolo 123 - 29/9/2000

Resumo: No Brasil, o complexo sucroalcooleiro ocupa lugar de destaque, com 5,4 milhões de hectares de cana colhida para a indústria em 2000, dos quais 2,5 milhões no Estado de São Paulo. O setor agrícola representa cerca de $65 \%$ dos custos na produção de álcool e açúcar e aproximadamente $45 \%$ desse custo são destinados à etapa de plantio. O baixo rendimento das operações de plantio onde um trabalhador planta, em média, 0,27 ha $\mathrm{d}^{-1}$, justifica o desenvolvimento de um mecanismo dosador de rebolos de cana-de-açúcar, tendo como objetivo a distribuição uniforme de mudas. Utilizando-se recursos computacionais de engenharia como WM3D e MatLab, procurou-se aprimorar um dispositivo de dosagem de rebolos existente. O modelo otimizado, quando simulado com apenas um dos diâmetros de rebolo em análise, apresentou resultados satisfatórios e características compatíveis às dos modelos que apresentaram condição ideal de operação. As simulações do modelo otimizado para variações do diâmetro do rebolo, da ordem de $10 \mathrm{~mm}$ apresentaram, como resposta, incidência de células com excesso de rebolo, resposta esta também verificada nos resultados experimentais.

Palavras-chave: Saccharum officinarum, plantio, simulação dinâmica

\section{Improvement of the billet metering mechanism used for mechanical planting of sugarcane}

\begin{abstract}
Brazilian production of alcohol and sugar holds an important position among producing countries, with 5.4 million hectares of sugarcane harvested by the industry during 2000 . The State of São Paulo contributes with 2.5 million hectares of this total. The agricultural operations represent approximately $65 \%$ of the total sugar and alcohol production costs. Nearly $45 \%$ of the agricultural operational cost is due to planting. The low yield for conventional planting operations

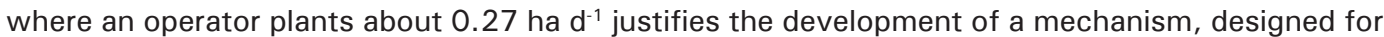
uniform plant distribution. The objective of this work was to improve the performance of an existing sugarcane planter, using computational engineering aids such us WM3D and MatLab. The optimized model simulated with only one of the analyzed sugarcane diameters, showed satisfactory results, with characteristics compatible with the models presenting ideal operational conditions. Optimized model simulations for cane diameter varying by $10 \mathrm{~mm}$ showed cells filled with excess of billets, which is in agreement with the experimental results.
\end{abstract}

Key words: Saccharum officinarum, planting, dynamic simulation

\section{INTRODUÇÃO}

O complexo sucroalcooleiro ocupa lugar de destaque no agronegócio paulista, produzindo açúcar e álcool combustível, além de inúmeros outros produtos de relevância para a economia nacional. Trata-se de setor historicamente marcado pela intervenção governamental direta, incluindo o próprio planejamento da produção, mas que nos anos recentes passa por profundas mudanças no aparato regulatório, consistindo de um setor em franco processo de ajustamento às novas condições de concorrência (Gonçalves \& Veiga Filho, 1999).

O setor canavieiro enfrenta problemas pela falta de tecnologia apropriada, principalmente no setor agrícola que, aliado ao problema da sazonalidade da mão-de-obra, tem onerado o custo da produção o qual, sem o subsídio do Governo Federal, tem enfrentado sérios problemas financeiros (Magalhães \& Braunbeck, 1998). Na produção de álcool e açúcar, o setor agrícola representa cerca de $65 \%$ do custo total e apresenta maior disponibilidade na redução dos custos e, dentro dele, uma fatia 
significativa do custo, aproximadamente $45 \%$, é destinada à operação de plantio (FNP, 1999).

O desenvolvimento de um mecanismo dosador de rebolos de cana-de-açúcar para o plantio mecanizado é cada vez mais necessário, uma vez que existe o interesse de se desenvolver um implemento que proporcione distribuição uniforme, evitando falhas de deposição de mudas, ou seja, realize um plantio de boa qualidade com menor quantidade de cana-semente, resultando em economia de mudas. Esses fatores, associados, geram maior rendimento, pois elevam a capacidade de plantio, aproveitando melhor as condições climáticas e reduzindo o custo de produção.

Com este objetivo Dias Neto \& Magalhães (1997) construíram e avaliaram um dispositivo dosador de rebolos em laboratório. Embora o mecanismo tenha apresentado uma série de falhas, os resultados mostraram o seu potencial.

O presente trabalho teve como objetivo principal aprimorar o dosador de rebolos de cana-de-açúcar, utilizando-se recursos de engenharia, de modo a viabilizá-lo para futura aplicação em campo.

\section{MATERIAL E MÉTODOS}

Para o desenvolvimento deste projeto foi realizada, inicialmente, uma análise dinâmica do dispositivo original, com o programa computacional $\mathrm{WM}^{3} \mathrm{D}^{1}$ identificando as variáveis de projeto que influenciam no desempenho do dispositivo.

O WM3D é um programa de simulação dinâmica tridimensional, que opera em computadores desktop em ambiente Windows, calculando o movimento de corpos por meio de técnicas de interação numérica. Este recurso possibilita a construção de sistemas complexos, permitindo calcular-se o movimento dos corpos sujeitos a restrições e forças. Além de restrições impostas pelo usuário como, por exemplo, sistema mola/amortecedor, articulações, este programa realiza simulações de interações multi-corpo.

Os fatores analisados durante esta simulação dinâmica foram: incidência de células sem rebolo (vazios); incidência de células com um rebolo (simples); incidência de células com dois rebolos (duplos) e incidência de células com três rebolos (triplos).

A partir dos resultados obtidos por meio do WM3D, elaborou-se um conjunto de equações não lineares, que foram resolvidas utilizando-se a função fsolve do $\mathrm{MatLab}^{2}$, cuja resposta gerou uma função objetivo, a qual foi otimizada com o auxílio da função constr do MatLab.

Com os resultados do modelo otimizado, foi possível elaborar-se um novo modelo para simulação virtual no WM3D, contendo 18 rebolos, com os diâmetros de: 20,25 e $30 \mathrm{~mm}$, com 6 rebolos de cada diâmetro (modelo Otimizado 1); 20 e $25 \mathrm{~mm}$, com 9 rebolos de cada diâmetro (modelo Otimizado 2); 25 e $30 \mathrm{~mm}$, com 9 rebolos de cada diâmetro (modelo Otimizado 3); 20 e $30 \mathrm{~mm}$, com 9 rebolos de cada diâmetro (modelo Otimizado 4). Em todos eles, a velocidade tangencial adotada para o cilindro dosador foi de $0,19 \mathrm{~m} \mathrm{~s}^{-1}$. O modelo otimizado também foi submetido a variações do ângulo de inclinação da calha de

Programa desenvolvido pela MSC Software, para simulação dinâmica de corpos rígidos ${ }^{2}$ Programa desenvolvido pela MathWorks Inc, para computação numérica alimentação e caixa armazenadora $\left(40^{\circ}\right.$ e $\left.50^{\circ}\right)$ a fim de se verificar qual a influência deste fator na dosagem de rebolos.

\section{Avaliação do modelo otimizado}

A partir do resultado do modelo otimizado, o protótipo estacionário do mecanismo dosador foi projetado e construído no Laboratório de Protótipos do Departamento de Máquinas Agrícolas, enquanto a análise de resultados da unidade dosadora de rebolos de cana-de-açúcar foi realizada utilizando-se parâmetros, como: diâmetro médio dos rebolos, número de células vazias e quantidade de células preenchidas com mais de um rebolo.

À unidade dosadora foi acionada por uma unidade hidrostática de velocidade variável desenvolvida por Braunbeck \& Magalhães (Gonçalves, 1996). Este sistema de acionamento permite obter velocidades de rotação que variam de 50 até $1500 \mathrm{~min}^{-1}$, com um torque máximo de $500 \mathrm{Nm}$. Como a unidade dosadora deve operar a velocidades de rotação de 3 a $5,4 \mathrm{~min}^{-1}$, para satisfazer as condições impostas na simulação virtual, utilizou-se um redutor de engrenagens.

\section{Diâmetro dos rebolos}

Para se determinar o diâmetro médio dos rebolos, foram feitas três medições por rebolo, um em cada internódio localizado nas proximidades de cada extremidade, e outro na gema, localizada nas proximidades da metade do rebolo e as mudas de cana-de-açúcar utilizadas foram fornecidas pela Copersurcar, unidade experimental de Piracicaba.

\section{Condições de operação}

Utilizaram-se os seguintes parâmetros para a análise do desempenho da unidade dosadora: $(\mathrm{C} 1)$ : rebolos preenchendo apenas a região de interface do cilindro dosador e boca de descarga. Nesta região e sobre a calha de alimentação, foram depositados de 15 a 20 rebolos de cana; (C2): rebolos preenchendo $50 \%$ da capacidade da caixa armazenadora; (C3): rebolos preenchendo $100 \%$ da capacidade da caixa armazenadora.

Velocidade de rotação do cilindro dosador: $3,1,4,2$ e 5,4 $\mathrm{min}^{-1}$, denominadas R1, R2 e R3, respectivamente, sendo cada uma combinada a cada tipo de capacidade da caixa armazenadora; estabeleceu-se, após testes preliminares, o tempo de $5 \mathrm{~s}$, para que a unidade dosadora entre em regime de funcionamento. Os testes tiveram duração de $10 \mathrm{~s}$, realizando-se 10 repetições.

Os dados obtidos foram analisados por meio da ferramenta Análise de Dados - Anova: fator duplo com repetição, do Excel. Esta opção realiza uma análise estatística de blocos casualizados com 2 fatores com repetição.

\section{CONSIDERAÇÕES GERAIS}

O aprimoramento da unidade dosadora parte da identificação das variáveis de projeto que, muitas vezes, são dependentes umas das outras.

Associando-se a velocidade de trabalho (V), número de rebolos plantado por metro linear (n), rotações do cilindro dosador (ROT) e número de células do cilindro dosador $(\mathrm{N})$, chega-se à relação: 


$$
\mathrm{ROT}=\frac{\mathrm{Vn}}{\mathrm{N} 0,06}
$$

\section{Velocidade de trabalho do trator}

Considera-se que a unidade dosadora a ser desenvolvida realize, de forma concomitante, as etapas básicas de plantio de cana-de-açúcar: abertura dos sulcos, colocação de fertilizantes, distribuição de colmos e fechamento dos sulcos; portanto, a velocidade de trabalho ideal para as condições impostas está em torno de $4 \mathrm{~km} \mathrm{~h}^{-1}$.

\section{Característica dos rebolos para a simulação virtual}

Utilizaram-se 18 rebolos de $380 \mathrm{~mm}$, para cada simulação.

$\mathrm{O}$ fator diâmetro do rebolo foi analisado separadamente, ou seja, em cada simulação foi utilizado um único diâmetro de rebolo. Os diâmetros analisados foram: 20, 25 e $30 \mathrm{~mm}$, com massa de 118, 184 e $266 \mathrm{~g}$, respectivamente, e os rebolos foram considerados uniformes, com o centro de massa localizado no centro geométrico do corpo, sendo o coeficiente de atrito de 0,73 entre rebolos e 0,59 entre rebolos e aço, coeficiente de restituição de 0,01 ; foram admitidas três gemas por rebolo, o que corresponde a quatro rebolos a serem plantados por metro linear, desconsiderando-se o índice de curvatura.

\section{Boca de descarga}

A abertura da boca de descarga da caixa armazenadora de rebolo, foi dimensionada de maneira que permitisse o fluxo de rebolo entre a caixa armazenadora e a calha de alimentação. Iznaga (1997) observou que arranjos com três mudas na boca de descarga apresentam instabilidade, o que garante o escoamento e, para arranjos com menos de três mudas na boca de descarga, apresentaram estabilidade, situação que impede o escoamento.

\section{Ponto de inserção e ângulo de inclinação da calha de alimentação}

O ponto de inserção, já havia sido determinado por Dias Neto \& Magalhães (1997) estando situado a $20^{\circ}$ do eixo X em relação ao centro do cilindro dosador. $\mathrm{O}$ ângulo de inclinação foi de $30^{\circ} \mathrm{em}$ relação ao eixo $\mathrm{X}$, fator este só variado durante as simulações virtuais realizadas no modelo otimizado, pois sua variação está relacionada, segundo os autores, com:

- o aumento da camada de rebolo na região de interface entre a boca de descarga e o cilindro dosador, para ângulos maiores que $30^{\circ}$;

- as falhas de abastecimento, devido ao atrito entre os rebolos e a calha de alimentação, para ângulos menores que $30^{\circ}$.

\section{Diâmetro do cilindro dosador}

Os raios totais (raio do cilindro + altura da talisca) utilizados foram: 0,$155 ; 0,2325 ; 0,310$ e $0,465 \mathrm{~m}$, que correspondem: a 1 ; 1,$5 ; 2$ e 3 vezes, respectivamente, ao raio do cilindro dosador desenvolvido por Dias Neto \& Magalhães (1998).

\section{Velocidade de rotação}

Tendo-se a velocidade tangencial (Vt) limite de $0,19 \mathrm{~m} \mathrm{~s}^{-1}$ determinada experimentalmente por Dias Neto \& Magalhães
(1997) calcularam-se, a partir da Eq. (2) as seguintes rotações (ROT): $12,8,6$ e $4 \mathrm{~min}^{-1}$, para os raios (R) de 0,$155 ; 0,2325 ; 0,310$ e $0,465 \mathrm{~m}$, respectivamente.

$$
\mathrm{Vt}=\frac{\mathrm{ROT}}{60} 2 \pi \mathrm{R}
$$

\section{Altura e espaçamento entre taliscas}

Foram analisados três diâmetros para o rebolo de canasemente, a saber: 15,25 e $30 \mathrm{~mm}$. A altura de $30 \mathrm{~mm}$ corresponde à altura utilizada no protótipo desenvolvido por Dias Neto \& Magalhães (1998) sendo que a de $15 \mathrm{~mm}$ corresponde a 50\% desta altura e a de $25 \mathrm{~mm}$ ao diâmetro médio da cana, adotado para o desenvolvimento da unidade dosadora.

Os espaçamentos utilizados foram 26 e $34 \mathrm{~mm}$, em que este corresponde ao espaçamento utilizado no protótipo desenvolvido por Dias Neto \& Magalhães (1998); já o espaçamento de $26 \mathrm{~mm}$ foi adotado em conseqüência do diâmetro médio considerado, acrescido de $1 \mathrm{~mm}$, para propiciar bom acomodamento do rebolo na célula do cilindro dosador. Valores inferiores a $26 \mathrm{~mm}$ foram descartados, pois dificultariam a acomodação de rebolos com diâmetro superior a $25 \mathrm{~mm}$ nas células.

\section{TESTES PRELIMINARES}

\section{Simulação Inicial}

Para efetuar-se a simulação do dispositivo desenvolvido por Dias Neto \& Magalhães (1998) apresentado na Figura 1, foi necessário estabelecer-se os seguintes critérios e hipóteses:

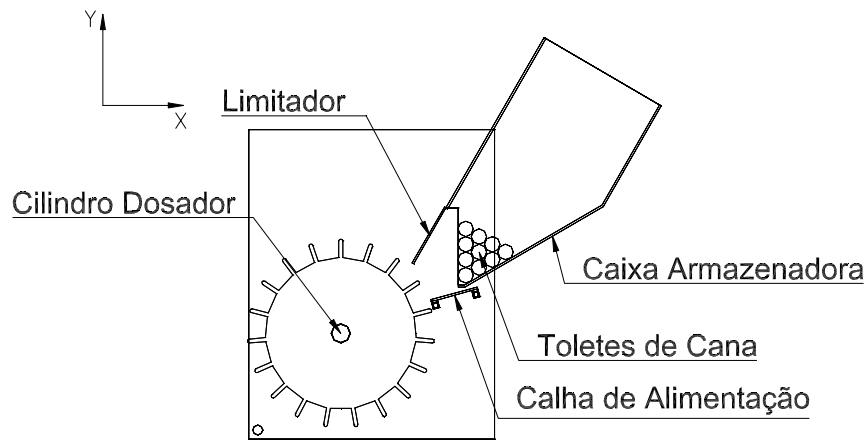

Figura 1. Modelo 1 (Dias Neto \& Magalhães, 1998)

1. Caixa armazenadora: o ângulo de inclinação utilizado foi de $30^{\circ}$ em relação ao eixo X. Abertura da boca de descarga corresponde a $124,5 \mathrm{~mm}$ e o coeficiente de atrito ${ }^{3}$ entre aço e cana-de-açúcar é de 0,59 , enquanto o coeficiente de restituição é de 0,01 . Como restrição ao movimento, a caixa está ancorada em relação ao sistema de coordenadas global;

2. Rebolos de cana-de-açúcar: para a simulação, foram considerados 10 rebolos com $25 \mathrm{~mm}$ de diâmetro $0,38 \mathrm{~m}$ de comprimento e o coeficiente de atrito entre as canas 0,73 ; o coeficiente de restituição é de 0,01 ;

O coeficiente de atrito representa uma propriedade de interação entre dois objetos. No Working Model 3D, cada corpo possui seu coeficiente de atrito. O coeficiente de atrito entre dois objetos é determinado tomando-se o menor valor dos coeficientes dos corpos em contato. Por exemplo, o coeficiente de atrito do corpo 1 é 0,05 e do corpo 2 é 0,3 , portanto o coeficiente de atrito a ser utilizado quando os dois corpos estivem em contato será de 0,05 
3. Calha de alimentação: o ângulo de inclinação utilizado foi de $15^{\circ} \mathrm{em}$ relação ao eixo X. Coeficiente de atrito 0,59 . Coeficiente de restituição 0,01 . Como restrição ao movimento, ancorou-se o corpo em relação ao sistema de coordenadas global;

4. Lateral: coeficiente de atrito 0,59 . coeficiente de restituição 0,01 . O corpo foi ancorado em relação ao sistema de coordenadas global.

5. Cilindro dosador: diâmetro total de $0,31 \mathrm{~m}$, comprimento de $0,4 \mathrm{~m}$, espaçamento entre taliscas de $34 \mathrm{~mm}$ e altura das taliscas $30 \mathrm{~mm}$. Coeficiente de atrito 0,59 e de restituição 0,01 . Como restrições ao movimento do corpo no espaço, foi inserido um motor de revolução, com 8 rotações $\min ^{-1}$ de velocidade angular, situado no centro de massa do corpo, responsável pela rotação do cilindro dosador.

6. Limitador: coeficiente de atrito 1,10 e de restituição 0,02 . A restrição imposta para o movimento do corpo foi uma junta de revolução mola/amortecedor, inserida na região de contato entre o limitador e a caixa armazenadora. Esta junta representa a função de flexibilidade do limitador, pois o WM3D considera todas as peças como corpos rígidos. A junta age como uma mola de torsão em torno do eixo Z, possibilitando, assim, maior ou menor descarga de rebolo sobre o cilindro dosador. Os coeficientes utilizados foram:

a) Equação da mola:

$$
\mathrm{T}=-\mathrm{kx}^{2}
$$

onde:

$\mathrm{T}$ - torque aplicado $(\mathrm{N} \mathrm{mm})$

$\mathrm{k}$ - coeficiente de rigidez $\left(\mathrm{N} \mathrm{mm} \mathrm{deg}^{-2}\right)$

x - deslocamento angular da mola (graus).

$$
\mathrm{k}=5\left(\frac{\mathrm{Nmm}}{\mathrm{deg}^{2}}\right)
$$

b) Equação do amortecedor:

$$
\mathrm{F}=-\mathrm{c} \mathrm{x}
$$

onde:

$$
\begin{array}{ll}
\mathrm{F} & \text { - força aplicada }(\mathrm{N}) \\
\mathrm{c} & \text { - coeficiente de amortecimento }\left(\mathrm{N} \mathrm{mm} \mathrm{s} \mathrm{deg}^{-1}\right) \text {. }
\end{array}
$$

$$
\mathrm{c}=0,914\left(\frac{\mathrm{N} \mathrm{mm} \mathrm{s}}{\operatorname{deg}}\right)
$$

7. Controle Integrador: A técnica de integração utilizada foi o método de Euler, com passo de integração de $0,01 \mathrm{~s}$ (fixo). As configurações de tolerância para posição, orientação, sobreposição e entre as peças, foram: $1 \mathrm{~mm}, 1^{\circ}, 0,75$ e $0,1 \mathrm{~mm}$, respectivamente.

\section{Variáveis de projeto}

Por meio de modificações de alguns parâmetros no Modelo 1, associadas a novas simulações, identificaram-se parâmetros fundamentais para o desenvolvimento de um modelo, a fim de que ele opere em condições ideais. As variáveis encontradas foram: espaçamento entre taliscas (E); altura da talisca $(\mathrm{H})$; diâmetro do cilindro dosador $(\Phi)$; abertura da boca de descarga da caixa armazenadora (A); distância da boca de descarga em relação ao cilindro dosador (D); ponto de inserção da caixa de descarga e da calha de alimentação em relação ao cilindro dosador (P) e inclinação da caixa armazenadora e da calha de alimentação $(\beta)$.

\section{SIMULAÇÃO E OTIMIZAÇÃO}

\section{Condições de simulação}

Foram gerados 24 modelos (Tabela 1), a partir da variação dos parâmetros H, E e R.

Tabela 1. Descrição dos modelos submetidos à simulação virtual

\begin{tabular}{cccccccc}
\hline Modelos & $\mathrm{H}$ & $\mathrm{E}$ & $\mathrm{R}$ & $\mathrm{N}$ & $\begin{array}{c}\mathrm{ROT} \\
(\mathrm{rpm})\end{array}$ & $\begin{array}{c}\mathrm{v} \\
\left(\mathrm{km} \mathrm{h}^{-1}\right)\end{array}$ & $\begin{array}{c}\mathrm{Vt} \\
\left(\mathrm{m} \mathrm{s}^{-1}\right)\end{array}$ \\
\cline { 2 - 4 } 1 & 15 & 26,1 & 155 & 27 & 12 & 4,9 & 0,19 \\
2 & 25 & 26,2 & 155 & 25 & 12 & 4,5 & 0,19 \\
3 & 30 & 26,3 & 155 & 24 & 12 & 4,3 & 0,19 \\
4 & 15 & 33,5 & 155 & 22 & 12 & 4,0 & 0,19 \\
5 & 25 & 34,3 & 155 & 20 & 12 & 3,6 & 0,19 \\
6 & 30 & 34,8 & 155 & 19 & 12 & $3, .4$ & 0,19 \\
19 & 15 & 26,1 & 232,5 & 42 & 8 & 5,0 & 0,19 \\
20 & 25 & 26,2 & 232,5 & 40 & 8 & 4,8 & 0,19 \\
21 & 30 & 26,2 & 232,5 & 39 & 8 & 4,7 & 0,19 \\
22 & 15 & 33,6 & 232,5 & 34 & 8 & 4,1 & 0,19 \\
23 & 25 & 34,3 & 232,5 & 32 & 8 & 3,8 & 0,19 \\
24 & 30 & 34,6 & 232,5 & 31 & 8 & 3,7 & 0,19 \\
7 & 15 & 26,1 & 310 & 57 & 6 & 5,1 & 0,19 \\
8 & 25 & 26,1 & 310 & 55 & 6 & 5,0 & 0,19 \\
9 & 30 & 26,2 & 310 & 54 & 6 & 4,9 & 0,19 \\
10 & 15 & 33,9 & 310 & 46 & 6 & 4,1 & 0,19 \\
11 & 25 & 34,3 & 310 & 44 & 6 & 4,0 & 0,19 \\
12 & 30 & 33,6 & 310 & 44 & 6 & 4,0 & 0,19 \\
13 & 15 & 26,1 & 420 & 87 & 4 & 5,2 & 0,19 \\
14 & 25 & 26,1 & 420 & 85 & 4 & 5,1 & 0,19 \\
15 & 30 & 26,1 & 420 & 84 & 4 & 5,0 & 0,19 \\
16 & 15 & 34 & 420 & 70 & 4 & 4,2 & 0,19 \\
17 & 25 & 34,2 & 420 & 68 & 4 & 4,1 & 0,19 \\
18 & 30 & 33,8 & 420 & 68 & 4 & 4,1 & 0,19 \\
\hline
\end{tabular}

Onde: $\mathrm{H}$ - altura da talisca; $\mathrm{E}$ - espaçamento entre talisacas; $\mathrm{R}$ - raio total do cilindro dosador; $\mathrm{N}$ - número de células do cilindro dosador; ROT - rotação do cilindro daosador; $\mathrm{v}$ - velocidade linear; Vt - velocidade tangencial do cilindro dosador

Os modelos foram submetidos à simulação virtual, utilizando-se as seguintes condições de controle de integração

- Integrador: Euler

- Integração

a) Passo de Integração: $0,0005 \mathrm{~s}$

b) Passos por Quadro: 2

- Configuração de tolerância
a) Posição: $1 \mathrm{~mm}$
b) Orientação: $1^{\circ}$
c) Tolerância de sobreposição: 0,01
d) Tolerância entre as peças: $0,1 \mathrm{~mm}$
e) Dígitos significativos: 5

\section{Otimização}

Com os resultados dos modelos obtidos por meio do WM3D, elaboraram-se 24 equações para cada um dos três diâmetros de rebolo em análise, cada qual correspondendo a 
um modelo específico, sendo que cada equação continha 24 variáveis obtidas a partir de $\mathrm{H}, \mathrm{E}$ e $\mathrm{R}$ e suas combinações. O termo independente de cada equação é representado pelo número incidente de células contendo um rebolo.

Equação genérica:

$$
\begin{aligned}
\mathrm{f}_{\mathrm{i}}=0 & \mathrm{H}_{\mathrm{i}} * \mathrm{x}_{1}+\mathrm{E}_{\mathrm{i}} * \mathrm{x}_{2}+\mathrm{R}_{\mathrm{i}} * \mathrm{x}_{3}+\mathrm{H}_{\mathrm{i}} * \mathrm{E}_{\mathrm{i}} * \mathrm{x}_{4}+ \\
& \mathrm{H}_{\mathrm{i}} * \mathrm{R}_{\mathrm{i}} * \mathrm{x}_{5}+\mathrm{E}_{\mathrm{i}} * \mathrm{R}_{\mathrm{i}} * \mathrm{x}_{6}+\mathrm{H}_{\mathrm{i}} * \mathrm{E}_{\mathrm{i}} * \mathrm{R}_{\mathrm{i}} * \mathrm{x}_{7}+ \\
& \mathrm{H}_{\mathrm{i}}{ }^{2} * \mathrm{x}_{8}+\mathrm{E}_{\mathrm{i}}{ }^{2} * \mathrm{x}_{9}+\mathrm{R}_{\mathrm{i}}{ }^{2} * \mathrm{x}_{10}+\mathrm{H}_{\mathrm{i}}{ }^{2} * \mathrm{E}_{\mathrm{i}} * \mathrm{x}_{11}+ \\
& \mathrm{H}_{\mathrm{i}}{ }^{2} * \mathrm{R}_{\mathrm{i}} * \mathrm{x}_{12}+\mathrm{H}_{\mathrm{i}} * \mathrm{E}_{\mathrm{i}}{ }^{2} * \mathrm{x}_{13}+\mathrm{E}_{\mathrm{i}}{ }^{2} * \mathrm{R}_{\mathrm{i}} * \mathrm{x}_{14}+ \\
& \mathrm{H}_{\mathrm{i}} * \mathrm{R}_{\mathrm{i}}{ }^{2} \mathrm{x}_{15}+\mathrm{E}_{\mathrm{i}} * \mathrm{R}_{\mathrm{i}}{ }^{2} * \mathrm{x}_{16}+\mathrm{H}_{\mathrm{i}}{ }^{2} * \mathrm{E}_{\mathrm{i}} * \mathrm{R}_{\mathrm{i}} * \mathrm{x}_{17}+ \\
& \mathrm{H}_{\mathrm{i}} * \mathrm{E}_{\mathrm{i}}{ }^{2} * \mathrm{R}_{\mathrm{i}} * \mathrm{x}_{18}+\mathrm{H}_{\mathrm{i}} * \mathrm{E}_{\mathrm{i}} * \mathrm{R}_{\mathrm{i}}{ }^{2} * \mathrm{x}_{19}+ \\
& \mathrm{H}_{\mathrm{i}}{ }^{2} * \mathrm{E}_{\mathrm{i}}{ }^{2} * \mathrm{x}_{20}+\mathrm{H}_{\mathrm{i}}{ }^{2} * \mathrm{R}_{\mathrm{i}}{ }^{2} * \mathrm{x}_{21}+\mathrm{E}_{\mathrm{i}}{ }^{2} * \mathrm{R}_{\mathrm{i}}{ }^{2} * \mathrm{x}_{22}+ \\
& \mathrm{H}_{\mathrm{i}}{ }^{2} * \mathrm{E}_{\mathrm{i}}{ }^{2} * \mathrm{R}_{\mathrm{i}} * \mathrm{x}_{23}+\mathrm{H}_{\mathrm{i}}{ }^{2} * \mathrm{E}_{\mathrm{i}} * \mathrm{R}_{\mathrm{i}}{ }^{2} * \mathrm{x}_{24}-\mathrm{S}_{\mathrm{i}}
\end{aligned}
$$

Elaboradas as equações, obteve-se um sistema não-linear, Eq. (8) para cada diâmetro de rebolo analisado:

$$
F_{i}=\left[\begin{array}{l}
f_{1} \\
f_{2} \\
\vdots \\
f_{24}
\end{array}\right]
$$

O sistema não-linear foi resolvido com auxilio do MatLab, utilizando-se a função fsolve do módulo Optimization Toll.Box, obtidos, como resposta, os valores dos coeficientes $\mathrm{x}_{1}, \ldots, \mathrm{x}_{24}$. De posse desses coeficientes, montou-se uma equação que representa o conjunto de equações $\mathrm{f}_{\mathrm{i}}$, utilizando-se como base a Eq. (4) - em que H, E e R, correspondem a x(1), x(2) e x(3) respectivamente, representada por:

$$
\begin{aligned}
\mathrm{t}_{\mathrm{i}}= & \mathrm{x}_{1} * \mathrm{x}(1)+\mathrm{x}_{2} * \mathrm{x}(2)+\mathrm{x}_{3} * \mathrm{x}(3)+\mathrm{x}_{4} * \mathrm{x}(1) * \mathrm{x}(2)+ \\
& \mathrm{x}_{5} * \mathrm{x}(1) * \mathrm{x}(3)+\mathrm{x}_{6} * \mathrm{x}(2) * \mathrm{x}(3)+ \\
& \mathrm{x}_{7} * \mathrm{x}(1) * \mathrm{x}(2) * \mathrm{x}(3)+\mathrm{x}_{8} * \mathrm{x}(1)^{2}+\mathrm{x}_{9} * \mathrm{x}(2)^{2}+ \\
& \mathrm{x}_{10} * \mathrm{x}(3)^{2}+\mathrm{x}_{11} * \mathrm{x}(1)^{2} * \mathrm{x}(2)+\mathrm{x}_{12} * \mathrm{x}(1)^{2} * \mathrm{x}(3)+ \\
& \mathrm{x}_{13} * \mathrm{x}(1) * \mathrm{x}(2)^{2}+\mathrm{x}_{14} * \mathrm{x}(2)^{2} * \mathrm{x}(3)+\mathrm{x}_{15} * \mathrm{x}(1) * \mathrm{x}(3)^{2}+ \\
& \mathrm{x}_{16} * \mathrm{x}(2) * \mathrm{x}(3)^{2}+\mathrm{x}_{17} * \mathrm{x}(1)^{2} * \mathrm{x}(2) * \mathrm{x}(3)+ \\
& \mathrm{x}_{18} * \mathrm{x}(1) * \mathrm{x}(2)^{2} * \mathrm{x}(3)+\mathrm{x}_{19} * \mathrm{x}(1) * \mathrm{x}(2) * \mathrm{x}(3)^{2}+ \\
& \mathrm{x}_{20} * \mathrm{x}(1)^{2} * \mathrm{x}(2)^{2}+\mathrm{x}_{21} * \mathrm{x}(1)^{2} * \mathrm{x}(3)^{2}+\mathrm{x}_{22} * \mathrm{x}(2)^{2} * \mathrm{x}(3)^{2}+ \\
& \mathrm{x}_{23} * \mathrm{x}(1)^{2} * \mathrm{x}(2)^{2} * \mathrm{x}(3)+\mathrm{x}_{24} * \mathrm{x}(1)^{2} * \mathrm{x}(2) * \mathrm{x}(3)^{2}
\end{aligned}
$$

A partir da Eq. (9) efetuou-se a otimização para cada condição de diâmetro de rebolo utilizado na simulação virtual, cujos parâmetros de análise são compostos pelo conjunto de variáveis - H, E e R, representado por x(1), x(2) e x(3), respectivamente, utilizados para a elaboração dos modelos.

O processo de otimização foi realizado com o auxilio do MatLab, por meio da função constr, otimizando uma variável de cada vez, ou seja, adotando-se valores para duas variáveis e se deixando a Eq. (9) em função de apenas uma variável.

A primeira variável a ser otimizada foi $\mathrm{x}(3)$ - raio total do cilindro dosador, e os valores adotados para $\mathrm{x}(1)$ e $\mathrm{x}(2)$ - altura da talisca e espaçamento entre taliscas, foram os do início do intervalo dos parâmetros analisados, 15 e $26 \mathrm{~mm}$, respectivamente, chegando-se, assim, à seguinte equação para a otimização de R:

$$
\begin{aligned}
\mathrm{t}_{\mathrm{i}}= & \mathrm{x}_{1} * 15+\mathrm{x}_{2} * 26+\mathrm{x}_{3} * \mathrm{x}(3)+\mathrm{x}_{4} * 390+ \\
& \mathrm{x}_{5} * 15 * \mathrm{x}(3)+\mathrm{x}_{6} * 26 * \mathrm{x}(3)+\mathrm{x}_{7} * 390 * \mathrm{x}(3)+ \\
& \mathrm{x}_{8} * 225+\mathrm{x}_{9} * 676+\mathrm{x}_{10} * \mathrm{x}(3)^{2}+\mathrm{x}_{11} * 5850+ \\
& \mathrm{x}_{12} * 225 * \mathrm{x}(3)+\mathrm{x}_{13} * 10140+\mathrm{x}_{14} * 676 * \mathrm{x}(3)+ \\
& \mathrm{x}_{15} * 15 * \mathrm{x}(3)^{2}+\mathrm{x}_{16} * 26 * \mathrm{x}(3)^{2}+\mathrm{x}_{17} * 5850 * \mathrm{x}(3)+ \\
& \mathrm{x}_{18} * 10140 * \mathrm{x}(3)+\mathrm{x}_{19} * 390 * \mathrm{x}(3)^{2}+\mathrm{x}_{20} * 152100+ \\
& \mathrm{x}_{21} * 225 * \mathrm{x}(3)^{2}+\mathrm{x}_{22} * 676 * \mathrm{x}(3)^{2}+\mathrm{x}_{23} * 152100 * \mathrm{x}(3)+ \\
& \mathrm{x}_{24} * 5850 * \mathrm{x}(3)^{2}
\end{aligned}
$$

Para a resolução da equação anterior foi necessário inserir-se as seguintes restrições:

$$
\begin{gathered}
\mathrm{g}(1)_{\mathrm{i}}=\mathrm{x}_{1} * 15+\mathrm{x}_{2} * 26+\mathrm{x}_{3} * \mathrm{x}(3)+\mathrm{x}_{4} * 390+ \\
\mathrm{x}_{5} * 15 * \mathrm{x}(3)+\mathrm{x}_{6} * 26 * \mathrm{x}(3)+\mathrm{x}_{7} * 390 * \mathrm{x}(3)+ \\
\mathrm{x}_{8} * 225+\mathrm{x}_{9} * 676+\mathrm{x}_{10} * \mathrm{x}(3)^{2}+\mathrm{x}_{11} * 5850+ \\
\mathrm{x}_{12} * 225 * \mathrm{x}(3)+\mathrm{x}_{13} * 10140+\mathrm{x}_{14} * 676 * \mathrm{x}(3)+ \\
\mathrm{x}_{15} * 15 * \mathrm{x}(3)^{2}+\mathrm{x}_{16} * 26 * \mathrm{x}(3)^{2}+\mathrm{x}_{17} * 5850 * \mathrm{x}(3)+ \\
\mathrm{x}_{18} * 10140 * \mathrm{x}(3)+\mathrm{x}_{19} * 390 * \mathrm{x}(3)^{2}+\mathrm{x}_{20} * 152100+ \\
\mathrm{x}_{21} * 225 * \mathrm{x}(3)^{2}+\mathrm{x}_{22} * 676 * \mathrm{x}(3)^{2}+\mathrm{x}_{23} * 152100 * \mathrm{x}(3)+ \\
\mathrm{x}_{24} * 5850 * \mathrm{x}(3)^{2}-18 \\
\mathrm{~g}(2)=155-\mathrm{x}(3) \\
\mathrm{g}(3)=-465+\mathrm{x}(3)
\end{gathered}
$$

A Eq. (11) indica que a Eq. (10) assume 18 como o máximo valor permitido, pois este foi o número de rebolos utilizado para cada ensaio, enquanto as Eqs. (12) e (13) representam o intervalo utilizado na simulação virtual; conseqüentemente, o mínimo e máximo valor que x(3) poderá assumir.

O valor encontrado de $\mathrm{x}(3)$ foi utilizado como valor adotado para a otimização da próxima variável; no nosso caso, $x(2)$, na Eq. (9), foi mantido o valor de x(1) adotado para a otimização de $\mathrm{x}(3)$. Os valores otimizados de x(2) e x(3) foram, então, utilizados como valores para a otimização de $\mathrm{x}(1)$. Repetiu-se o processo a partir da otimização de x(3) agora com novos valores de x(1) e $\mathrm{x}(2)$ até que houvesse estabilização dos resultados, isto é, quando se constatou, ao se terminar a otimização de x(1), que o valor encontrado combinado com o valor de $\mathrm{x}(2)$ do passo anterior, já havia sido utilizado para a otimização de $\mathrm{x}(3)$.

As restrições para x(2) e x(1), são respectivamente:

$$
\begin{gathered}
g(2)=26-x(2) \\
g(3)=-35+x(2) \\
g(2)=15-x(1) \\
g(3)=-30+x(1)
\end{gathered}
$$

A resposta da otimização foi um conjunto composto por $\mathrm{x}(1), \mathrm{x}(2)$ e $\mathrm{x}(3)$ otimizados, correspondentes a H, E e R, para 
cada um dos diâmetros de rebolo analisados. Este conjunto de dados foi comparado aos resultados obtidos por meio da simulação virtual, para determinação de sua validade. A etapa seguinte foi a otimização das três variáveis em estudo concomitante, para determinação de que conjunto de dados otimizados para determinado diâmetro de rebolo é válido para os demais diâmetros de rebolo em análise, utilizando-se, para tal, as Eqs. (9), (12), (13) e (14). O conjunto inicialmente adotado, $\mathrm{x} 0$, representa o resultado das variáveis otimizadas para um diâmetro de rebolo. Quando a resposta obtida, das equações acima, é igual ao valor $\mathrm{x} 0$, sabe-se que o modelo atende também às condições impostas pelo outro diâmetro de rebolo.

\section{RESULTADOS E DISCUSSÃO}

\section{Simulação virtual}

A partir dos 24 modelos virtuais do dosador propostos e desenhados conforme descrito na Tabela 1, obtiveram-se os resultados de incidência de rebolo nas células, conforme apresentado na Tabela 2.

Tabela 2. Incidência de rebolos

\begin{tabular}{|c|c|c|c|c|c|c|c|c|c|c|c|c|c|}
\hline \multirow{3}{*}{ Modelos } & \multicolumn{13}{|c|}{ Diâmetro dos Rebolos (mm) } \\
\hline & \multicolumn{4}{|c|}{20} & \multicolumn{5}{|c|}{25} & \multicolumn{4}{|c|}{30} \\
\hline & $\mathrm{V}$ & $\mathrm{S}$ & $\mathrm{D}$ & $\mathrm{T}$ & Q & V & $\mathrm{S}$ & $\mathrm{D}$ & $\mathrm{T}$ & V & $\mathrm{S}$ & $\mathrm{D}$ & $\mathrm{T}$ \\
\hline 1 & - & 14 & 2 & - & - & - & 8 & 5 & - & - & 7 & 4 & 1 \\
\hline 2 & - & 12 & 3 & - & - & - & 16 & 1 & - & - & 14 & 2 & - \\
\hline 3 & - & 3 & - & 5 & - & - & 6 & 6 & - & - & 12 & 3 & - \\
\hline 4 & - & 1 & 4 & 3 & - & - & 3 & 3 & 2 & - & 12 & 3 & - \\
\hline 5 & - & 3 & 2 & 1 & 2 & - & 5 & 5 & 1 & - & 8 & 5 & - \\
\hline 6 & - & 1 & - & 3 & 2 & - & 4 & 1 & 4 & - & 12 & 3 & - \\
\hline 19 & - & 12 & 3 & - & - & - & 14 & 2 & - & - & 18 & - & - \\
\hline 20 & - & 13 & 1 & 1 & - & - & 18 & - & - & - & 16 & 1 & - \\
\hline 21 & - & 2 & 3 & 2 & 1 & - & 10 & 4 & - & - & 18 & - & - \\
\hline 22 & - & 5 & 5 & 1 & - & - & 7 & 1 & 3 & - & 12 & 3 & - \\
\hline 23 & - & 10 & 1 & 2 & - & - & 12 & 3 & - & - & 18 & - & - \\
\hline 24 & - & 5 & - & 3 & 1 & - & 8 & 2 & 2 & - & 18 & - & - \\
\hline 7 & - & 18 & - & - & - & - & 18 & - & - & - & 18 & - & - \\
\hline 8 & - & 16 & 1 & - & - & - & 18 & - & - & - & 18 & - & - \\
\hline 9 & - & 1 & 7 & 1 & - & - & 18 & - & - & - & 18 & - & - \\
\hline 10 & - & 12 & - & 2 & - & - & 8 & 2 & 2 & - & 14 & 2 & - \\
\hline 11 & - & 18 & - & - & - & - & 18 & - & - & - & 18 & - & - \\
\hline 12 & - & 1 & 1 & 5 & - & - & 18 & - & - & - & 18 & - & - \\
\hline 13 & - & 18 & - & - & - & - & 18 & - & - & - & 18 & - & - \\
\hline 14 & - & 18 & - & - & - & - & 18 & - & - & - & 18 & - & - \\
\hline 15 & - & 9 & 3 & 1 & - & - & 18 & - & - & - & 18 & - & - \\
\hline 16 & - & 16 & 1 & - & - & - & 18 & - & - & - & 18 & - & - \\
\hline 17 & - & 18 & - & - & - & - & 18 & - & - & - & 18 & - & - \\
\hline 18 & - & 6 & - & 4 & - & - & 18 & - & - & - & 18 & - & - \\
\hline
\end{tabular}

Onde: V - incidência de células vazias; $\mathrm{S}$ - incidência de células contendo 1 rebolo; D - incidência de células contendo 2 rebolos; T - incidência de células contendo 3 rebolos; Q - incidência de células contendo 4 rebolos

O número de rebolo para a simulação virtual se restringe a 18 rebolos, devido à incapacidade de processamento do WM3D em sistemas dinâmicos com presença de muitos corpos livres, o que torna o sistema complexo, à medida que os corpos começam a interagir com outros corpos ou peças que compõem o sistema. O resultado desta condição é um número elevado de equações de movimento geradas, pois para cada corpo livre são atribuídas três equações de movimento compromentendo, portanto, a capacidade operacional do programa.
A análise da Tabela 2 indica que, para:

1. Rebolos de $20 \mathrm{~mm}$ de diâmetro: cinco modelos apresentaram os melhores resultados, que podem ser divididos em dois grupos, o primeiro com altura da talisca igual a $15 \mathrm{~mm}$ e espaçamento entre taliscas de $26 \mathrm{~mm}$ - modelos 7 e 11 , e o segundo com altura da talisca igual a $25 \mathrm{~mm}$ e espaçamento entre elas de $34 \mathrm{~mm}$, modelos 13, 14 e 17. Todos esses modelos tinham raio do cilindro dosador superior a $0,31 \mathrm{~m}$. Pode-se observar que para espaçamento entre taliscas de $34 \mathrm{~mm}$, quando combinados com altura de talisca igual a $15 \mathrm{~mm}$ e as combinações de altura de talisca de $30 \mathrm{~mm}$ com quaisquer espaçamentos entre taliscas, resultam em áreas propícias à acomodação de mais de um rebolo por célula.

2. Rebolos de $25 \mathrm{~mm}$ de diâmetro: os melhores resultados ocorreram para o caso onde o raio total do cilindro dosador é superior a $0,31 \mathrm{~m}$, independente das combinações de espaçamento entre taliscas com altura de taliscas, com exceção da combinação de altura da talisca de $15 \mathrm{~mm}$ com espaçamento de $34 \mathrm{~mm}$, pois esta configuração resultou em áreas propícias à acomodação de mais de um rebolo por célula.

3. Rebolos de $30 \mathrm{~mm}$ de diâmetro: os resultados desejáveis começaram a surgir com raio total do cilindro dosador superior a $0,2325 \mathrm{~m}$. Com exceção do modelo 16 , assim como para rebolos de diâmetros de 20 e $25 \mathrm{~mm}$, as combinações de altura da talisca igual a $15 \mathrm{~mm}$ e espaçamento entre elas igual a $34 \mathrm{~mm}$, apresentaram resultados com incidência de rebolo em excesso.

\section{Otimização}

Os resultados obtidos pela otimização, apresentados nas Tabelas 3, 4 e 5, foram substituídos nas Eqs. (9), (12), (13) e (14) a fim de realizar-se a otimização concomitante das três variáveis em estudo, com a finalidade de se determinar que conjunto de dados otimizados para determinado diâmetro, é válido para os demais; por exemplo, na verificação do conjunto de dados otimizados para diâmetros de rebolo de $20 \mathrm{~mm}$, os valores encontrados nas Tabela 3 (A, B e C) são adotados como valor inicial $(\mathrm{x} 0)$ para a otimização das equações dos rebolos com diâmetro de 25 e $30 \mathrm{~mm}$; o mesmo foi realizado para a verificação dos demais resultados otimizados, e as respostas obtidas a partir desta verificação podem ser observadas na Tabela 4 (A, B e C).

Tabela 3. Resultados otimizados para rebolos de diferentes diâmetros

\begin{tabular}{|ccccccc|}
\hline \multicolumn{3}{|c}{ Valor Inicialmente Adotado } & & \multicolumn{4}{c|}{ Resultado da Otimização } \\
\hline $\mathrm{x}(1)$ & $\mathrm{x}(2)$ & $\mathrm{x}(3)$ & & $\mathrm{H}$ & $\mathrm{E}$ & $\mathrm{R}$ \\
\hline A. $20 \mathrm{~mm}$ & & & & & & \\
15 & 26 & & & & 264,8851 \\
15 & & 264,8851 & & 26 & \\
\hline
\end{tabular}

\section{B. $25 \mathrm{~mm}$}

\begin{tabular}{|c|c|c|c|c|c|}
\hline \multirow{2}{*}{$\begin{array}{l}15 \\
15\end{array}$} & 26 & & & \multirow[b]{2}{*}{26} & \multirow[t]{2}{*}{345,0905} \\
\hline & 26 & $\begin{array}{l}345,0905 \\
3450905\end{array}$ & 15 & & \\
\hline \multicolumn{6}{|c|}{ C. $30 \mathrm{~mm}$} \\
\hline 15 & 26 & & & \multirow{3}{*}{26} & 239,8369 \\
\hline 15 & & 239,8369 & & & \\
\hline & 26 & 239,8369 & 15 & & \\
\hline
\end{tabular}

Obs.: Quadros hachurados representam a variável que está sendo otimizada, onde: x(1) - altura da talisca $(\mathrm{mm}) ; \mathrm{x}(2)$ - espaçamento entre taliscas $(\mathrm{mm}) ; \mathrm{x}(3)$ - raio total do cilindro dosador $(\mathrm{mm})$; $\mathrm{H}$ - altura da talisca $(\mathrm{mm}) ; \mathrm{E}$ - espaçamento entre talisacas $(\mathrm{mm}) ; \mathrm{R}$ - raio total do cilindro dosador $(\mathrm{mm})$ 
Tabela 4. Resposta dos resultados otimizados de rebolos com diferentes diâmetros

\begin{tabular}{lcccccc}
\hline \multicolumn{5}{c}{ X0 } & & \multicolumn{3}{c}{ Resposta } \\
\cline { 1 - 3 } x $(1)$ & $\mathrm{x}(2)$ & $\mathrm{x}(3)$ & & $\mathrm{H}$ & $\mathrm{E}$ & $\mathrm{R}$ \\
\hline A. $20 \mathrm{~mm}$ & & & & & \\
15 & 26 & 264,8851 & 15 & 26 & 264,8851 \\
15 & 26 & 345,0905 & 15 & 26,0829 & 345,0904 \\
15 & 26 & 239,8369 & $16,0808^{*}$ & 26 & 239,8768 \\
B. $25 \mathrm{~mm}$ & & & & & \\
15 & 26 & 264,8851 & 15,0105 & 26,2121 & 264,8858 \\
15 & 26 & 345,0905 & 15 & 26 & 345,0904 \\
15 & 26 & 239,8369 & 15,0158 & 26,2614 & 239,8377 \\
C. $30 \mathrm{~mm}$ & & & & & \\
15 & 26 & 264,8851 & 15,5482 & 26,0042 & $253,9353^{*}$ \\
15 & 26 & 345,0905 & 15 & 26,0661 & 345,0905 \\
15 & 26 & 239,8369 & 15 & 26 & 239,8369 \\
\hline
\end{tabular}

A condição de x0 que apresentou melhor comportamento, foi a obtida a partir do conjunto otimizado para rebolos de $25 \mathrm{~mm}$ de diâmetro, pois este não apresentou variações significativas na resposta para a otimização concomitante realizada nos rebolos de diâmetro de 20 e $30 \mathrm{~mm}$, enquanto o modelo otimizado que foi submetido à análise de simulação virtual terá, como características, os valores expressos na Tabela 5.

Tabela 5. Características do modelo otimizado

\begin{tabular}{ccccccc}
\hline $\mathrm{H}$ & $\mathrm{E}$ & $\begin{array}{c}\mathrm{R} \\
\mathrm{mm}\end{array}$ & $\mathrm{N}$ & $\begin{array}{c}\mathrm{ROT} \\
(\mathrm{m})\end{array}$ & $\begin{array}{c}\mathrm{V} \\
\left(\mathrm{km} \mathrm{h}^{-1}\right)\end{array}$ & $\begin{array}{c}\mathrm{Vt} \\
\left(\mathrm{m} \mathrm{s}^{-1}\right)\end{array}$ \\
\hline 15 & 26 & 0,345 & 64 & 5,4 & 5,2 & 0,19 \\
\hline
\end{tabular}

$\mathrm{H}$ - altura da talisca; E - espaçamento entre taliscas; $\mathrm{R}$ - raio total do cilindro dosador; $\mathrm{N}$ - número de células do cilindro dosador; $\mathrm{v}$ - velocidade linear; $\mathrm{Vt}$ - velocidade tangencial do cilindro dosador

\section{Simulação virtual do modelo otimizado:}

A incidência de rebolos por célula para as condições impostas ao modelo otimizado está apresentada na Tabela 6 .

A partir dos resultados apresentados na Tabela 6 observa-se, para a combinação de rebolos de vários diâmetros em uma simulação, que:

- variações de diâmetro de até $5 \mathrm{~mm}$ não promovem a ocorrência de rebolo em excesso, ou seja, incidência de duplos ou triplos. Portanto, as combinações de diâmetro utilizadas no modelo otimizado 2 (rebolos com diâmetro de 20 e $25 \mathrm{~mm}$ ) e otimizado 3 (rebolos com diâmetro de 25 e $30 \mathrm{~mm}$ ) não apresentam problema na dosagem, pois só é verificada a incidência de células com um rebolo;

- variações de diâmetro superiores a $5 \mathrm{~mm}$ ocasionam a incidência de rebolo em excesso nas células, caso dos modelos otimizado 1 (rebolos com diâmetro de 20, 25 e $30 \mathrm{~mm}$ ) e otimizado 4 (rebolos com diâmetro de 20 e $30 \mathrm{~mm}$ ).

Normalmente, ocorre concentração de rebolo na região de interface Caixa armazenadora, calha de alimentação e cilindro dosador, possibilitando que alguns rebolos caminhem certa parte do percurso em excesso nas células; quando um ou dois rebolos de $20 \mathrm{~mm}$ estão em excesso em uma célula que já acomoda um rebolo de $20 \mathrm{~mm}$ de diâmetro e esta célula está localizada à frente de uma célula que, por sua vez, acomoda um rebolo de $30 \mathrm{~mm}$ de diâmetro, ocorre a incidência de excesso de rebolo na célula, condição em que o rebolo de $30 \mathrm{~mm}$ age como obstáculo, impedindo os rebolos de $20 \mathrm{~mm}$ em excesso de escoarem para a região de interface, pois estes não têm energia potencial suficiente para vencer o obstáculo gerado pelo rebolo de $30 \mathrm{~mm}$ acomodado.

No modelo otimizado foi verificado, também, a influência do ângulo de inclinação da calha de descarga na unidade dosadora; para tanto, variou-se o ângulo de inclinação de $30^{\circ}$ para $40^{\circ}$ e $50^{\circ}$ em relação ao eixo $\mathrm{X}$, obtendo-se os resultados apresentados na Tabela 6 .

A análise desses dados indica aumento de incidência de células com duplos e triplos, à medida que o ângulo de inclinação aumenta. Esta tendência é explicada devido ao aumento da altura da camada de rebolos na região de interface cilindro dosador - boca de descarga.

\section{Desempenho da unidade dosadora}

Nos 487 rebolos utilizados no ensaio, o diâmetro médio encontrado foi de $26,9 \mathrm{~mm}$, com desvio-padrão de $2,7 \mathrm{~mm}$ e o número médio de gemas por rebolo foi de 3,9, com desvio-padrão de 1,1.

A análise dos dados, utilizando-se o esquema fatorial $3 \times 3$, para a incidência de rebolos nas células, demonstrou efeito significativo a 0,05 de probabilidade para a interação capacidade da caixa $(C)$ x rotação $(\mathrm{R})$; foi necessário, então, realizar-se o desdobramento desses fatores, de duas maneiras:

- para se estudar o comportamento da capacidade da caixa armazenadora para cada velocidade de rotação do cilindro dosador;

- para se estudar o comportamento da velocidade de rotação do cilindro dosador dentro de cada capacidade da caixa armazenadora.

Como ferramenta estatística para esta análise, utilizou-se o teste de Tukey, em que os resultados obtidos estão apresentados na Tabela 7.

Tabela 7. Comparação das médias para percentagem de incidências de células vazias, com 1,2 e 3 rebolos

\begin{tabular}{lclcc}
\hline \multirow{2}{*}{ Configuração } & \multicolumn{4}{c}{ Méida* $^{*}$} \\
\cline { 2 - 5 } & Vazia & 1 Rebolo & 2 Rebolos & 3 Rebolos \\
\hline C1R1 & $3,8 \mathrm{~cd}$ & $93,1 \mathrm{a}$ & $3,1 \mathrm{de}$ & $0,0 \mathrm{~d}$ \\
C1R2 & $9,6 \mathrm{~b}$ & $89,8 \mathrm{ab}$ & $0,7 \mathrm{e}$ & $0,0 \mathrm{~d}$ \\
C1R3 & $20,9 \mathrm{a}$ & $77,7 \mathrm{c}$ & $1,3 \mathrm{e}$ & $0,0 \mathrm{~d}$ \\
C2R1 & $0,0 \mathrm{~d}$ & $86,6 \mathrm{abc}$ & $13,4 \mathrm{c}$ & $0,0 \mathrm{~d}$ \\
C2R2 & $0,5 \mathrm{~d}$ & $79,1 \mathrm{c}$ & $20,5 \mathrm{c}$ & $0,0 \mathrm{~d}$ \\
C2R3 & $5,3 \mathrm{c}$ & $82,8 \mathrm{bc}$ & $11,9 \mathrm{~cd}$ & $0,0 \mathrm{~d}$ \\
C3R1 & $0,0 \mathrm{~d}$ & $27,5 \mathrm{f}$ & $63,1 \mathrm{a}$ & $9,4 \mathrm{a}$ \\
C3R2 & $0,7 \mathrm{~d}$ & $37,7 \mathrm{e}$ & $55,1 \mathrm{ab}$ & $6,5 \mathrm{~b}$ \\
C3R3 & $1,0 \mathrm{~d}$ & $48,7 \mathrm{~d}$ & $46,6 \mathrm{~b}$ & $3,8 \mathrm{c}$ \\
\hline
\end{tabular}

* Médias seguidas pela mesma letra, na mesma coluna, não diferem pelo teste de Tukey a 0,05 de probabilidade

Vazia $\left(\Delta_{0,05}=4,1\right) ; 1$ Rebolo $\left(\Delta_{0,05}=9,8\right) ; 2$ Rebolos $\left(\Delta_{0,05}=9,71\right) ; 3$ Rebolos $\left(\Delta_{0,05}=2,1\right)$

Tabela 6. Incidência de rebolos por célula para o modelo otimizado conforme o ângulo de inclinação da caixa armazenadora

\begin{tabular}{|c|c|c|c|c|c|c|c|c|c|c|c|c|}
\hline Modelo & Vazios & Simples & Duplos & Triplos & Vazio & Simples & Duplos & Triplos & Vazio & Simples & Duplos & Triplos \\
\hline Ângulo da Caixa & \multicolumn{4}{|c|}{$30^{\circ}$} & \multicolumn{4}{|c|}{$40^{\circ}$} & \multicolumn{4}{|c|}{$50^{\circ}$} \\
\hline Otimizado 1 & - & 15 & - & 1 & - & 10 & 1 & 2 & - & 8 & 2 & 2 \\
\hline Otimizado 2 & - & 18 & - & - & - & 16 & 1 & - & - & 14 & 2 & - \\
\hline Otimizado 3 & - & 18 & - & - & - & 14 & 2 & - & - & 14 & 2 & - \\
\hline Otimizado 4 & - & 14 & 2 & - & - & 10 & 1 & 2 & - & 8 & 2 & 2 \\
\hline
\end{tabular}


A análise da Tabela 7 permite a identificação de C1R3, como pior configuração durante a realização dos ensaios experimentais na unidade dosadora, pois foi a configuração que apresentou maior incidência de células vazias comprometendo, portando, o desempenho do sistema. As configurações C2R1 e C3R1 proporcionaram menor incidência de células vazias, equivalentes às configurações C1R1, C3R3, C3R2 e C2R2.

Nota-se, que a configuração C1R1 é a que apresenta melhor resposta à incidência de células com um rebolo, sendo esta equivalente às configurações $\mathrm{C} 1 \mathrm{R} 2 \mathrm{e} \mathrm{C} 2 \mathrm{R} 1$; pode-se verificar, também, que a configuração C3R1 apresenta a pior incidência de células com incidência de um rebolo; já a configuração C3R1, influi de maneira negativa no desempenho da unidade dosadora, pois contribui com alta incidência de células com dois rebolos, sendo equivalente à configuração $\mathrm{C} 3 \mathrm{R} 2$, enquanto a configuração C1R2 foi a que melhores resultados apresentou para a incidência de células com dois rebolos no desempenho da unidade dosadora; esta configuração é equivalente às configurações C1R3 e C1R1.

Observa-se, ainda, que a configuração C3R3 apresenta resultado menos satisfatório para o desempenho da unidade dosadora, para a incidência de células com três rebolos. O resultado ideal para o desempenho da unidade dosadora, para a incidência de células com três rebolos, é dado pelas configurações C1R1, C2R1, C1R2, C2R2, C1R3, C2R3.

\section{CONCLUSÕES}

1. O WM3D mostrou-se limitado quanto à simulação de mecanismos dotados de corpos livres.

2. O incremento de corpos livres contribui para o aumento de equações de movimento, prejudicando ou impossibilitando a capacidade de processamento.

3. O modelo otimizado, quando simulado com apenas um dos diâmetros de rebolo em análise, apresentou resultados satisfatórios, e suas características são compatíveis com as dos modelos que apresentaram condição ideal de operação.

4. A variação, para ângulos superiores do ângulo de inclinação da calha de alimentação e caixa armazenadora do modelo otimizado, na simulação virtual, contribuiu para a incidência de células com excesso de rebolo.
5. As simulações do modelo otimizado para variações do diâmetro do rebolo da ordem de $10 \mathrm{~mm}$ apresentaram, como resposta, incidência de células com excesso de rebolo. Esta resposta também foi verificada nos resultados experimentais.

6. A configuração de operação C1R1 - calha de alimentação com rebolos e velocidade de rotação do cilindro dosador de $3,1 \mathrm{~min}^{-1}$, foi a que apresentou as melhores respostas para incidência de rebolo nas células.

7. A alimentação da caixa armazenadora deve ser realizada de forma contínua e uniforme, para configurações C1R2 e C1R5. Esta prática é importante para garantir a incidência de rebolo nas células.

\section{LITERATURA CITADA}

Dias Neto, A.F.; Magalhães, P.S.G. Mecanismo dosador de rebolos de cana-de-açúcar para plantio mecanizador. In: Congresso Interno de Iniciação Cientifica da UNICAMP, 5 , 1997, Campinas, Caderno de Resumos... Campinas: UNICAMP, 1997, p.117.

Dias Neto, A.F.; Magalhães, P.S.G. Desenvolvimento de um mecanismo dosador de rebolos de cana-de-açúcar. In: Congresso Brasileiro de Engenharia Agrícola, 27, 1998, Poços de Caldas. Anais... SBEA, v.III, 1998, p.157-159

FNP Consultoria \& Comércio. Cana-de-açúcar. In: Agrianual - 99, Anuário da Agricultura Brasileira, 1999. p.222-238.

Gonçalves, J.S.; Veiga Filho, A.A. Açúcar e álcool. www.iea.sp.gov.br/desem98.htm, 18 jan. 1999.

Gonçalves, M.A.D. Curvas de rendimento de uma unidade acionadora hidrostática de velocidade variável. Campinas: UNICAMP, 1996, 15p. Relatório final de estágio supervisionado

Iznaga, A.M.B. Ordenamento de mudas de cana-de-açúcar para a aplicação em plantadoras automáticas. Campinas: UNICAMP, 1997, 97p. Dissertação Mestrado

Magalhães, P.S.G.; Braunbeck, O.A. Colheita de cana-de-açúcar: atualidade e perspectiva. In: Balbuena, R.H.; Benez, S.H.; Joraruria, D. (eds.). Ingeneria Rural y Mecanización Agrária en el ámbito Latinoamericano. La Plata: Editora da la Universidad de La Plata, 1998, p.262-273. 